\title{
Controlling A Robotic Hip Exoskeleton with Noncontact Capacitive Sensors
}

\author{
Simona Crea, Member, IEEE, Silvia Manca, Andrea Parri, Enhao Zheng, Member, IEEE, Jingeng Mai, \\ Raffaele Molino Lova, Nicola Vitiello, Member, IEEE, and Qining Wang, Senior Member, IEEE
}

\begin{abstract}
For partial lower-limb exoskeletons, an accurate real-time estimation of the gait phase is paramount to provide timely and well-tailored assistance during gait. To this end, dedicated wearable sensors separate from the exoskeletons mechanical structure may be preferable because they are typically isolated from movement artifacts that often result from the transient dynamics of the physical human-robot interaction. Moreover, wearable sensors that do not require time-consuming calibration procedures are more easily acceptable by users. In this study a robotic hip orthosis was controlled using capacitive sensors placed in orthopedic cuffs on the shanks. The capacitive signals are zeroed after donning the cuffs and do not require any further calibration. The capacitive sensing-based controller was designed to perform online estimation of the gait cycle phase via adaptive oscillators, and to provide a phase-locked assistive torque. Two experimental activities were carried out to validate the effectiveness of the proposed control strategy. Experiments conducted with seven healthy subjects walking on a treadmill at different speeds demonstrated that the controller can estimate the gait phase with an average error of $4 \%$, while also providing hip flexion assistance. Moreover, experiments carried out with four healthy subjects showed that the capacitive sensing-based controller could reduce the metabolic expenditure of subjects compared to the unassisted condition (mean \pm SEM, $-\mathbf{- 3 . 2 \%} \pm \mathbf{1 . 1}$ ).
\end{abstract}

Index Terms - Capacitive sensing, gait phase estimation, wearable robotics, hip exoskeleton.

\section{INTRODUCTION}

W EARABLE robots have been developed with the aim of extending, complementing, substituting, or enhancing human motor functions by providing external mechanical

This work was supported by the EU within the CYBERLEGs Plus Plus project (H2020-ICT-2016-1 Grant Agreement \#731931), the National Natural Science Foundation of China (No. 91648207), the Beijing Natural Science Foundation (No. L182001) and the Beijing Municipal Science and Technology Project (No. Z181100009218007). (Corresponding author: Qining Wang)

S. Crea, S. Manca, A. Parri and N. Vitiello are with The BioRobotics Institute, Scuola Superiore SantAnna, Italy. S. Crea and N. Vitiello are also with Fondazione Don Gnocchi, Milan, Italy.

E. Zheng is with the State Key Laboratory of Management and Control for Complex Systems, Institute of Automation, Chinese Academy of Science, Beijing, China.

J. Mai is the Robotics Research Group, College of Engineering, Peking University, Beijing 100871, China and also with the Beijing Engineering Research Center of Intelligent Rehabilitation Engineering, Beijing 100871, China.

R. M. Lova is with Fondazione Don Carlo Gnocchi, Firenze, Italy.

Q. Wang is with the Robotics Research Group, College of Engineering, Peking University, Beijing, China and is also with the Beijing Innovation Center for Engineering Science and Advanced Technology (BIC-ESAT), China. (E-mail: qiningwang@pku.edu.cn)

S. Crea, A. Parri and N. Vitiello have commercial interests in IUVO S.r.l, a spin off company of Scuola Superiore SantAnna. Currently, the IP protecting the APO technology has been licensed to IUVO S.r.l. for commercial exploitation. S. Crea and S. Manca equally contributed to this work. N. Vitiello and Q. Wang equally contributed to this work. power to the user synchronously with his/her movements [1][6]. Wearable robots engage in a close physical and cognitive interaction with the user [7], [8]. The physical interaction has to be safe, comfortable, and ergonomic. Actuators are typically designed to be compliant, and the kinematic structure must feature mechanisms both for adjustable fitting to users with different anthropometries, and for the self-alignment of the robotic and human joint axes. Moreover, the cognitive human-robot interface needs to decode the human movement intention in real time in an intuitive way [7]-[10]. One major challenge for the cognitive interface of lower-limb assistive wearable robots is the on-line estimation of the cyclical phase of locomotion-related activities in a discrete or continuous fashion [11], to enable the synchronization of the robot action with the gait cycle in a time-independent fashion.

Although movement sensors integrated into wearable robots (e.g. joint encoders or potentiometers) could be considered the easiest option for tracking the user's movement and estimating the gait phase, the human-robot interaction dynamics and soft tissue compression when the device works in assistive mode can diminish the fidelity of the sensor signals and render the real-time estimation of gait phase more challenging [12]. By contrast, using sensors which are not integrated into the robotic systems structure may improve the performance of the realtime phase estimation, but in order to be acceptable by users, sensors should be easily wearable and should not require timeconsuming calibration processes.

Within the state of the art, a variety of biosignals have been exploited for decoding movement intentions [13]. Surface electromyography (sEMG) has been widely adopted in wearable robotics as a measure of muscle activity [14]-[17], capacitance tomography has been used to measure muscle properties [18], inertial measurement units (IMUs) [19]-[21] and sensorized insoles have been used for measuring kinematic and kinetic variables [22]. All of these sensing modalities have been proven effective for the control of wearable devices, but in many cases the usability by end-users is limited by complex donning and calibration procedures, or by long-term degradation of sensor performance.

As an additional biosensing modality for motion tracking and intention detection, capacitive pressure sensors can measure cyclic variations in lower-limb muscle volume due to contraction and relaxation. In contrast to sEMG, they do not need specially trained personnel for the placement of electrodes, can be worn over clothes, and do not require computationally intensive real-time data processing [23], [24]. Moreover, due to the simplicity of their signal processing electronics, capacitive sensors can be relatively economical. 


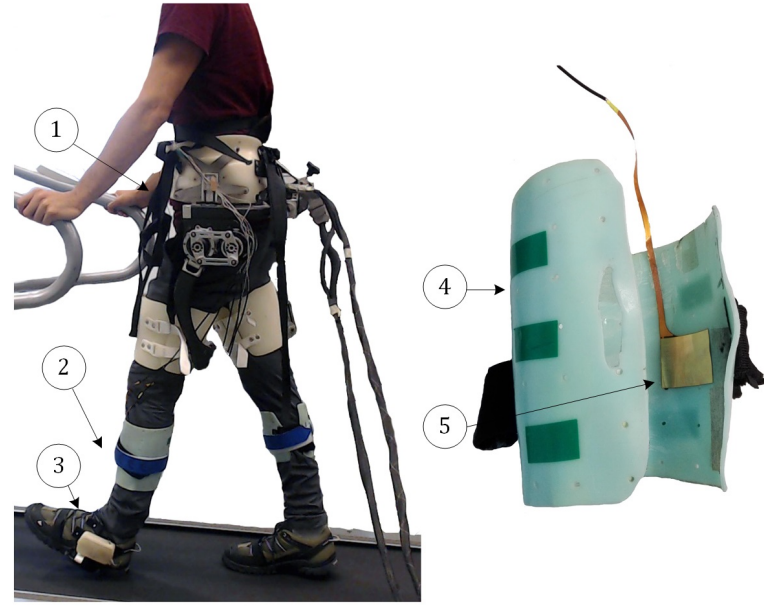

(a) (b)

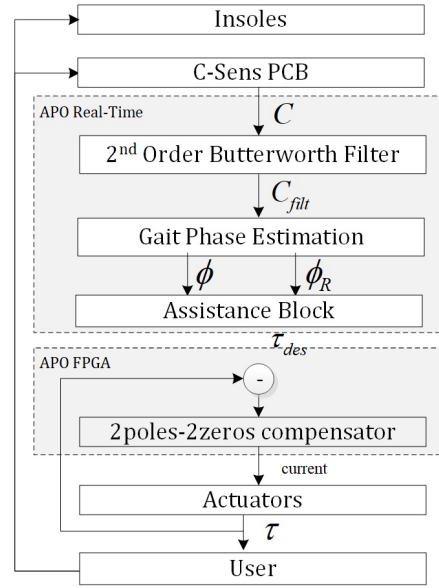

(c)

Fig. 1. Overview of the experimental setup and of the control architecture. (a) Participant wearing the (1) APO, (2) C-Sens cuffs and (3) shoes instrumented with sensorized insoles. (b) C-Sens cuff, made of an orthopaedic shell (4) and one electrode (5). The electrode is placed on the posterior side of the shank. The reference electrode is not shown. (c) Conceptual scheme of the control algorithm: the muscle contraction/relaxation measured from the C-Sens is sent to the APO control board. The filtered signal is used as input to the gait phase estimator and to the assistance block computing the desired torque reference. Insoles signals are not used for real-time control but are stored for offline analysis.

In this work, the feasibility of exploiting capacitive sensors to estimate the gait phase in real time and to control the assistive action of an active pelvis orthosis (APO, [25]) was investigated. In our previous study [26], the performance of gait phase estimation was validated offline using twelve capacitive sensors integrated into two cuffs - one placed on the thigh, and one on the shank. Capacitive signals were used as inputs for a gait phase estimator based on Adaptive Oscillators (AOs, [27]) and were demonstrated to be suitable for collecting movement information. In this study, a simplified sensor system was used, in which the shank cuff was equipped with only one capacitive sensor, whose output signal was used to estimate the gait phase in real time. Based on the estimated gait phase, the APO provided a phase-locked hip flexion torque. Two experiments with healthy individuals were performed to quantify the reliability and effectiveness of the controller. The reliability of the control strategy was tested by measuring the online gait phase estimation error, with seven healthy subjects walking on a treadmill at different walking speeds with the APO in both assisted and unassisted modes. The effectiveness of the developed control strategy was then verified by comparing the energy consumption of four healthy subjects walking with the APO in unassisted versus assisted modes, based on the developed controller.

The rest of this paper is organized as follows: the experimental setup and the assistive controller based on capacitive sensors are presented in Section II, together with the experimental protocol and data analysis techniques. Results are presented in Section III and discussed in Section IV. Section $\mathrm{V}$ draws the conclusion of the study.

\section{Materials And Methods}

\section{A. Experimental Setup}

The experimental setup was composed of two primary elements: the capacitive sensor (C-Sens) technology, and the
APO. An overview of the setup is given in Figure 1(a).

C-Sens is a wearable sensory device, developed at Peking University (Beijing, China), constituted by capacitive sensors [23] (Figure 1(b)). The device is composed of a custom printed circuit board (PCB) and two orthopaedic cuffs worn by the user on the distal lower-limb segments (shanks). In this experiment, each thermoplastic cuff (weight: approximately $300 \mathrm{~g}$ ) was instrumented with one flexible copper capacitive electrode placed on the inner posterior cuff surface (a simplified design from the one presented [26]), with a corresponding reference electrode placed directly in contact with the skin. A layer of silicone rubber ( $2.5 \mathrm{~mm}$ thickness) is placed above each sensor to electrically insulate them, and the electrodes are connected to the dedicated PCB by a wired connection. The C-Sens working principle is based on the measurement of the capacitance between the two plates (sensors). In this application, since the muscle volume varies during the gait cycle due to contractions and relaxations, the distance between the plates changes and results in a variation of the measured capacitance. More details about the technology and performance (e.g. testretest reliability) can be found in [26].

The APO was previously developed at Scuola Superiore Sant'Anna (Pisa, Italy) with the goal of providing powered assistance to hip flexion and extension during activities of daily living [25]. The device is built around a C-shaped carbon fiber frame, which surrounds the users hips and posterior pelvis, and physically interfaces to the trunk via three orthotic shells - two lateral and one rear. The back shell is fixed on a posterior bar and adjusted by a screw mechanism to provide ergonomic support of the lumbar region. The APO frame carries two actuation units, one on each side, employing a series elastic actuator architecture [28]. Each actuation unit is deployed about two parallel axes. The first axis is the output shaft of the DC motor coupled with the reduction stage and with an incremental encoder. The second axis is the actual 
flexion-extension axis of the robotic hip joint, which embeds a torsional spring (with a stiffness of $100 \mathrm{Nm} / \mathrm{rad}$ ), ensuring compliant interaction with the lower-limb segment. The two parallel axes of human and robot hip flexion-extension are connected by a 4-bar linkage mechanism and aligned to effect movement in the sagittal plane.

An absolute encoder measures the hip flexion-extension angle, which allows calculation of the deflection of the torsional spring, as the difference between the absolute encoder and the incremental encoder readings, which in turn allows the calculation of the output torque of the actuation unit, by applying the Hooks law. The output torque is then used to implement the closed-loop torque control. Two links are connected to the output of the flexion-extension axis of the actuation units. The two links, each featuring a passive rotational degree of freedom for hip abduction-adduction, physically interface with the user's thighs by means of orthotic shells. The soft orthotic material and the wide contact area of the shells serve to reduce and distribute the pressure on the user's skin. The entire system has a total weight of $4.2 \mathrm{~kg}$.

The APO control system runs on a real-time controller, (cRIO-9082, National Instruments, Austin, Texas, US), equipped with a $1.33 \mathrm{GHz}$ dual-core processor and a field programmable gate array (FPGA). A closed-loop torque controller based on a 2poles-2zeros compensator is responsible for tracking the desired assistive torque (i.e. reference torque) to be delivered to the user. The mechanics and closed-loop torque control performance of the APO are described in [25]. A high-level control layer calculates the desired torque profile. Notably, transparent mode (TM) corresponds to the unassisted use of the APO, namely when the desired torque is constant and set to $0 \mathrm{Nm}$.

In addition to the APO and C-Sens, a pair of instrumented shoes equipped with sensorized insoles was used as reference gait measurement system, in order to assess offline the performance of the gait phase estimator based on AOs and capacitive sensors. Each insole comprises 64 optoelectronic pressure transducers embedded into a flexible PCB and covered with a Silicone layer. When a compressive load is applied to the cover, the Silicone gradually deforms to occlude the light path between the emitter and the receiver, resulting in a variation of the output voltage [22], [29]. Each insole is connected to the dedicated electronic board by means of a ribbon cable and powered by a 3.7 V Li-Po battery. The data from all the transducers are sampled at $100 \mathrm{~Hz}$ and transmitted to the PC via a Bluetooth connection. The computation of the vertical ground reaction force (vGRF) and the longitudinal position of the centre of pressure $(\mathrm{CoP})$ is managed by the host PC in an online manner. The insoles weigh about $100 \mathrm{~g}$ each, accommodate European foot sizes 41-43, and were inserted into sneakers worn by the subject.

In the second experiment, an indirect calorimeter (Oxycon Mobile, Jaeger, Germany) was used to quantify the metabolic cost of walking.

\section{B. System Integration}

Capacitive signals are sent to the APO real-time control board by a serial RS232 connection. The APO high-level con- trol layer is responsible for (i) the acquisition and processing of capacitive signals, (ii) the gait phase estimation [27], and (iii) the assistance block (Figure 1(c)):

- Raw signals from left and right limbs, $C_{\text {raw }}$, are filtered by a second-order low-pass Butterworth filter, with a cutoff frequency of $1 \mathrm{~Hz}$;

- The filtered signal, $C_{f i l t}$, is used by the phase estimator block, which implements the AO-based gait phase estimator to track the gait phase $\phi$, with a two-step process, according to the method presented in [26]. First, AOs observe and estimate the phase of the periodic input signal; second, the detection of a desired event related to the input signal triggers the computation of the so-called phase reset error, $\phi_{R}$, which denotes the error between the estimated and desired phase at the moment of the event detection (ideally $0 \%$ of the stride). At steady state, the reset of the estimated gait phase $\phi$, i.e. the beginning of a new gait cycle, is locked with the desired event. In this study, the desired event is the occurrence of the maximum peak of the capacitive signal.

- The assistive torque profile $\tau_{d e s}(\phi)$, is then calculated as a function of the estimated gait phase $\phi$ :

$\tau_{\text {des }}(\phi)= \begin{cases}k\left(\phi_{R}\right) \cdot C_{\text {filt }}\left(\phi+\Delta_{\phi}\right), & \phi_{l}<\phi<\phi_{u} \& C_{\text {filt }}>0 \\ 0, & \text { elsewhere }\end{cases}$

where $C_{\text {filt }}$ is the filtered capacitive signal, $\phi_{l}$ and $\phi_{u}$ are the lower and the upper extremities of the phase window where the torque is delivered, $\Delta_{\phi}$ is a phase shift of the assistive torque and $k$ is the proportional gain, set by the experimenter to define the amplitude of the assistive torque. To reduce the intensity of the assistive torque when the gait phase is not properly tracked, i.e. when $\phi_{R} \gg 0 \mathrm{rad}$, the gain $k$ is set to its half value. Notably, $\Delta_{\phi}$ and $k\left(\phi_{R}\right)$ are manually adjusted for each participant, according to his/her preference.

\section{Experimental Protocol}

The study protocol was conducted at the premises of Fondazione Don Carlo Gnocchi (Florence, Italy). Experimental activities were approved by the local Ethics Committee, and all subjects signed a written informed consent before taking part to the experiments. The first experiment aimed to assess the performance of the C-Sens-based controller during treadmill walking. The second experiment aimed to evaluate the effectiveness of the proposed assistive strategy by measuring the metabolic cost of walking in assisted and unassisted conditions.

Before starting the experiment, each subject walked on the treadmill wearing the $\mathrm{C}$-Sens, the APO, and the instrumented shoes. C-Sens cuffs were worn on the shanks, outside the pants. The APO was used under transparent and assisted modes, to familiarize the subject with the system, adjust the assistance control parameters, and allow subjects to identify their preferred self-selected walking speed, $S_{N}$. Individual tuning of assistance parameters was accomplished during this preliminary session with the goal of providing assistance 
that preserves comfortable interaction. Subjective evaluation of comfort was considered the primary basis for tuning the assistive torque magnitude and timing.

1) Validation of the C-Sens-based controller: Subjects taking part in the first experiment were requested to perform two walking trials. In each trial, the subject walked on the treadmill for eleven minutes at three different speeds: $S_{N}$, a slow speed $\left(S_{S}\right)$, and a fast speed $\left(S_{F}\right)$, with $S_{S}$ and $S_{F}$ defined as $S_{N} \pm 0.8 \mathrm{~m} / \mathrm{s}$, respectively. The eleven minutes were structured as follows: $S_{S 1}, S_{N 1}, S_{F}, S_{N 2}, S_{S 2}$. The first condition, $S_{S}$, lasted three minutes, to ensure that the algorithm was properly synchronized with the user's gait; then each successive condition lasted 2 minutes.

The first trial was conducted with the APO set to TM, whereas the second trial consisted of the APO controlled in assistive mode (AM), running the C-Sens-based controller. A rest between the two trials allowed subjects to recover from potential fatigue. Seven healthy subjects (four males) participated to this experiment (age: $28.6 \pm 4.9$ years, weight $70.1 \pm 8.4 \mathrm{~kg}$, height $173.4 \pm 7.5 \mathrm{~cm})$. The average value of the self-selected speed, $S_{N}$, was $0.94 \pm 0.03 \mathrm{~m} / \mathrm{s}$.

2) Metabolic cost of walking: Subjects taking part in the second experiment were requested to wear the APO and walk on the treadmill at a self-selected speed for 12 minutes: during the first six minutes the APO was controlled in TM, and for the last six minutes the APO was switched to AM. The indirect calorimeter was used to measure the oxygen uptake $\left(V_{O 2}\right)$. Four healthy subjects volunteered in the experiments (three males, age: $26.8 \pm 0.9$ years, weight: $65.3 \pm 4.7 \mathrm{~kg}$, height: $173.8 \pm 4.8 \mathrm{~cm})$. The average value of the self-selected speed, $S_{N}$, was $0.97 \pm 0.06 \mathrm{~m} / \mathrm{s}$.

\section{Data Analysis}

Data were analyzed offline using Matlab (Mathworks, 2015b). The acquired signals, namely the capacitive signals, hip joint angles, and torques, were segmented into strides using the heel strike event detected by the insoles. In addition, for each stride, the APO power was computed offline. All variables were then interpolated into 100 samples, corresponding to $0-100 \%$ of the gait cycle. Averaged profiles and standard deviations were computed, to characterize the gait pattern and assistive action.

In order to assess the performance of the C-Sens-based phase estimator, the gait phase $(\phi)$ estimated online was compared to a reference phase, $\phi_{\text {Linear }}$, calculated offline as a linear function, from 0 to $100 \%$, between two consecutive peaks of the $C_{f i l t}$ signal [26], [27]. For each stride, the error $\left(\phi_{E r r}\right)$ between the estimated and reference gait phases was computed as the point-by-point difference of the two variables, from 0 to $100 \%$ of the gait cycle. For each stride, the root mean square of the phase error $\phi_{E r r}$, was estimated, namely the $\mathrm{RMSE}_{\phi_{E r r}}$. In addition, the phase reset error $\phi_{R}$ was computed for each stride, according to the definition reported in [27]. For each subject, $\mathrm{RMSE}_{\phi_{E r r}}$ was also calculated as the average across all strides. Reliability of the biomechanical event chosen as the reset of the estimated gait phase was also investigated by means of two parameters named $\Delta_{\phi_{C}}$ and $\Delta_{\phi_{\theta}}$.
They are defined as the difference of the gait phase at the heel strike (detected by the insoles) and, respectively, (i) at the capacitive peak, i.e. the event used for the phase reset by the real-time controller $\left(\Delta_{\phi_{C}}\right)$, and (ii) the maximum hip flexion peak $\left(\Delta_{\phi_{\theta}}\right)$, which was also used to reset the phase in [27].

For the first experiment, the aforementioned parameter$\mathrm{s}$ were extracted for both limbs and then averaged across subjects. Data were checked for normality by means of the Kolmogorov-Smirnov test, and then an inferential statistical test (paired Student's t-test) was conducted to assess for statistical differences in the performance of the gait phase estimation between the TM and the AM modalities.

For the second experiment, the oxygen uptake was normalized for the body mass and averaged in the last two minutes of TM and AM conditions. Five minutes of sitting baseline were recorded to compute the resting metabolic rate. Baseline values were subtracted from the two minutes at steady state in TM and AM. The variation of the metabolic rates (calculated via the Brockway equation) between the two conditions was computed. An inferential statistical test was conducted to assess differences between the two conditions.

\section{EXPERIMENTAL RESULTS}

\section{A. Experiment 1. Capacitive signals and assistive strategy}

The average capacitive signal along the gait cycle is presented in Figure 2(a) for a representative subject, for the three speed conditions $\left(S_{S}, S_{N}\right.$ and $\left.S_{F}\right)$, in TM and AM. The signal has a quasi-sinusoidal shape, with a maximum peak occurring at approximately $15 \%$ of the gait cycle after the heel strike for all speeds.

Figure 2(b) shows the average hip joint angles, torques, and powers in the TM and AM conditions for a representative subject in the first experiment. Hip joint angles showed an increase in the maximum hip flexion angle due to the assistive torque.

Results confirmed that the correlation between TM and AM is higher with respect to the capacitive signals versus hip joint angle. For the capacitive signals, the Pearson's correlation coefficients between the average profiles in TM and AM conditions were $0.98,0.99$, and 0.99 at natural, fast, and slow walking speeds, respectively - compared to $0.64,0.71$, and 0.62 at $S_{N}, S_{F}$, and $S_{S}$ for the hip angles.

In $\mathrm{AM}$, on average the assistive torque was delivered in the period between $35.9 \pm 7.6 \%$ and $84.9 \pm 5.8 \%$ of the stride, with $0 \%$ coinciding with the heel strike. The average torque peak across subjects was $5.90 \pm 1.40 \mathrm{Nm}$ in $S_{S}, 5.40 \pm 1.35 \mathrm{Nm}$ in $S_{N}$, and $5.02 \pm 1.47 \mathrm{Nm}$ in $S_{F}$. The average power peak across subjects was $16.83 \pm 4.82 \mathrm{~W}$ in $S_{S}, 18.10 \pm 4.92 \mathrm{~W}$ in $S_{N}$, and $17.63 \pm 4.64 \mathrm{~W}$ in $S_{F}$.

\section{B. Experiment 1. Performance of the C-Sens phase estimator}

Figure 3 illustrates the $\mathrm{RMSE}_{\phi_{E r r}}$ and $\mathrm{RMSE}_{\phi_{R}}$ in TM and AM for the three walking speeds. In TM, the across-subject average of the $\mathrm{RMSE}_{\phi_{E r r}}$ was $0.26 \pm 0.02 \mathrm{rad}$, corresponding to $4.4 \%$ of the stride phase, and the average $\mathrm{RMSE}_{\phi_{R}}$ was $0.26 \mathrm{rad}$ ( $4.0 \%$ of stride phase). In AM, the across-subject average of the $\mathrm{RMSE}_{\phi_{E r r}}$ was $0.27 \pm 0.03 \mathrm{rad}$ (4.7\% of stride 

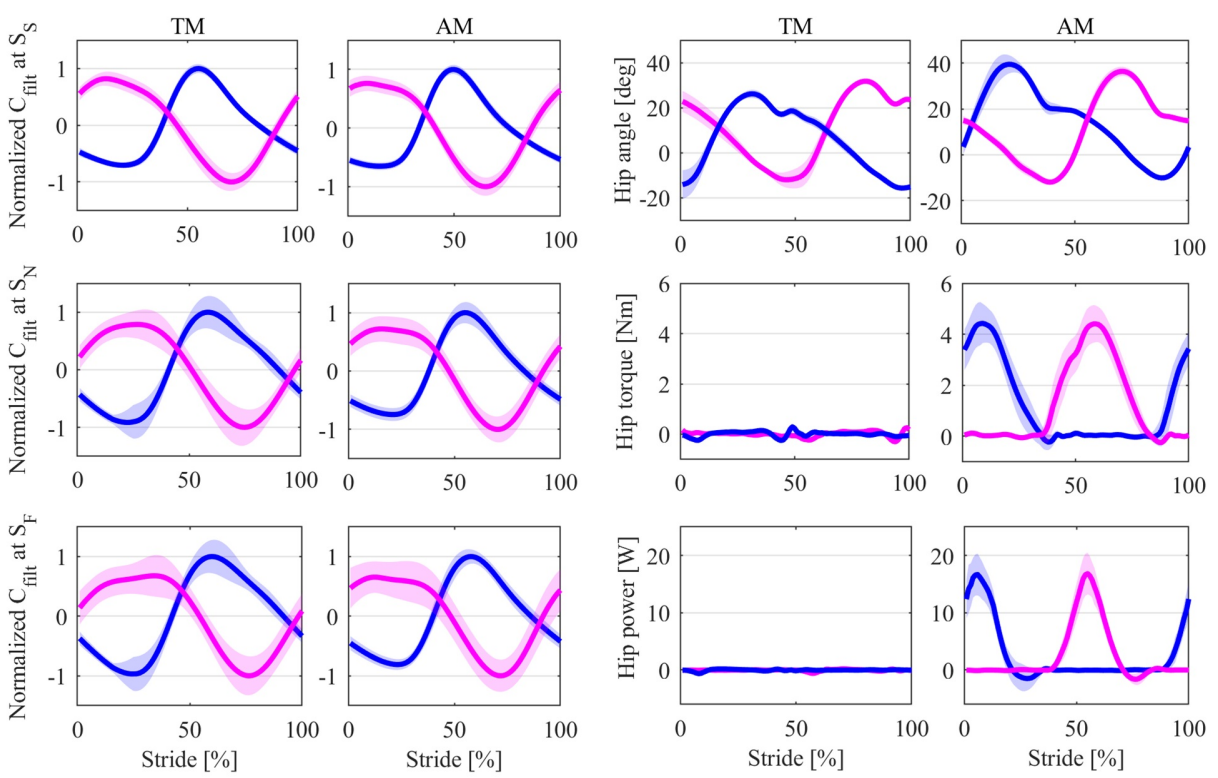

(a)
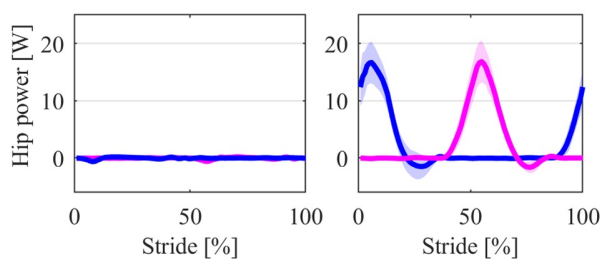

(b)

Fig. 2. Data from a representative subject in TM and AM modes. Data are segmented according to right heel strike detected with the insoles. The blue and pink plots show respectively the data from the left and right limb, contours represent the standard deviation for the three speed conditions, i.e. slow SS, normal SN and fast SF. (a) Normalized capacitive signals measured from the capacitive sensor positioned on the posterior side of the shank. (b) Kinematic and kinetic variables collected from the APO (hip joint angles, torque and power respectively). TM and AM refer to Transparent and Assistive Modes of the exoskeleton.

phase), and the average $\mathrm{RMSE}_{\phi_{R}}$ was $0.23 \mathrm{rad}$ (3.6\% of stride phase).

The normality of the data was verified by means of the Kolmogorov-Smirnov test (normal distributions $p=0.25$ and 0.49 for TM and AM, respectively). Then, the comparison of RMSE values between the TM and AM conditions revealed no statistically significant differences (paired Student's t-test, $p=0.73)$.

\section{Experiment 1. Phase Shift with respect to the heel strike}

The reliability of the resetting event was evaluated by comparing $\Delta_{\phi_{C}}$ and $\Delta_{\phi_{\theta}}$ (respectively) between TM and AM via paired Student's t-test. Across-subject average values and standard deviations are reported in Figure 4 for the three speeds. Results showed that both $\Delta_{\phi_{C}}$ and $\Delta_{\phi_{\theta}}$ significantly differ between TM and AM in all conditions, with a higher significance for $\Delta_{\phi_{\theta}} \mathrm{C}$ namely, $p=9.55 e-8<0.01$ for $\Delta_{\phi_{\theta}}$, and $p=0.022<0.05$ for $\Delta_{\phi_{C}}$.

\section{Experiment 2. Metabolic consumption}

For participants in the second experiment, the torque and power profiles averaged for each gait cycle and scaled for the subjects' body mass are shown in Figure 5. On average the assistive torque was delivered between $39.8 \pm 8.8 \%$ and $84.8 \pm 7.3 \%$ of the stride, with $0 \%$ coinciding with heel strike. The average across-subject peak torque was $5.22 \pm 0.65 \mathrm{Nm}$ and the average peak power was $19.26 \pm 5.91 \mathrm{~W}$. With respect to average peak power, $\mathrm{S} 10$ received the highest positive power $(0.38 \pm 0.01 \mathrm{~W} / \mathrm{kg})$, and $\mathrm{S} 8$ received the lowest positive power $(0.17 \pm 0.01 \mathrm{~W} / \mathrm{kg})$.

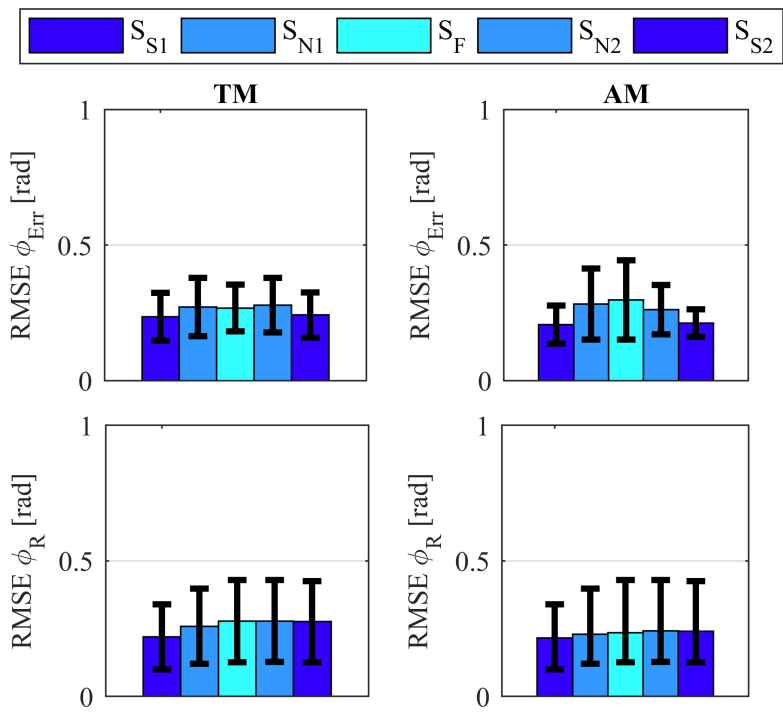

Fig. 3. (a) $\mathrm{RMSE}_{\phi_{E r r}}$ in TM condition (upper plots) and AM condition (bottom plots) in TM and AM conditions. Across-subjects averages and standard deviations are reported for all speeds (slow SS, normal SN and fast $\mathrm{SF})$. Note: $\phi_{E r r}$ is the error between the estimated and reference gait phases, calculated for each stride, as the point by point difference between the two variables. $\phi_{R}$ is the phase reset error computed for each stride, according to the definition reported in [27]. TM and AM refer to Transparent and Assistive modes of the exoskeleton. $\mathrm{RMSE}_{\phi_{E r r}}$, paired Student's t-test between TM and AM, $p=0.73$. 

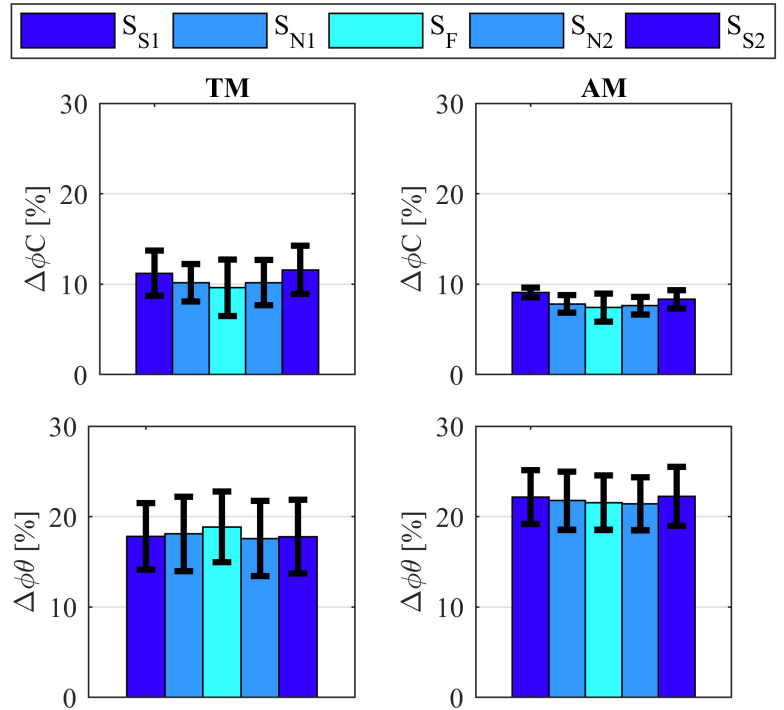

Fig. 4. $\Delta_{\phi} C$ (upper plots) and of $\Delta_{\phi} \theta$ (bottom plots) in TM and AM conditions. Across-subjects averages and standard deviations are reported for all speeds (slow SS, normal SN and fast SF). Note: $\Delta_{\phi} C$ is the difference of the gait phase at the heel strike and the capacitive peak, computed for each stride. $\Delta_{\phi} \theta$ is the difference of the gait phase at the heel strike and the maximum hip flexion peak. TM and AM refer to Transparent and Assistive modes of the exoskeleton. $\Delta_{\phi} \theta$, paired Student's t-test between TM and AM, $p=9.55 e-8<0.01 . \Delta_{\phi} C$ paired Student's t-test between TM and AM, $p=0.022<0.05$.

Figure 5 shows the average metabolic rate during sitting (BL), in TM, and AM. Subjects 8, 9, 10, and 11 showed reductions of $2.4 \%, 5.7 \%, 4.0 \%$, and $0.7 \%$ (respectively) in metabolic rate in AM relative to TM. A paired Student's t-test between TM and AM conditions showed a statistically significant reduction of the metabolic cost in $\operatorname{AM}(p=0.024<0.05)$ across all subjects.

\section{DISCUSSION}

Robustness and reliability of sensor signals are paramount for decoding the users intention and providing smooth and safe assistive action through lower-limb wearable robots. This work represents the first proof of feasibility that capacitive sensing techniques can be used for reliable measurement of gait-related motion signals and effective control of a robotic exoskeleton. The proposed sensing architecture features capacitive sensors placed on the shanks, providing quasi-sinusoidal signals related to muscle volume changes during locomotion. Such signals are used in real time to estimate the phase of the gait cycle, with the main advantages that they provide reliable and repeatable signals, which are not affected by the mechanical power transmitted by the hip exoskeleton when controlled in assistive mode. Despite this benefit, capacitive signals may also have disadvantages: for instance, here higher standard deviations at higher velocities were reported, probably caused by a less stable fit of the sensor cuffs on the shanks. In addition, sensitivity to electromagnetic fields can potentially cause signal deterioration.
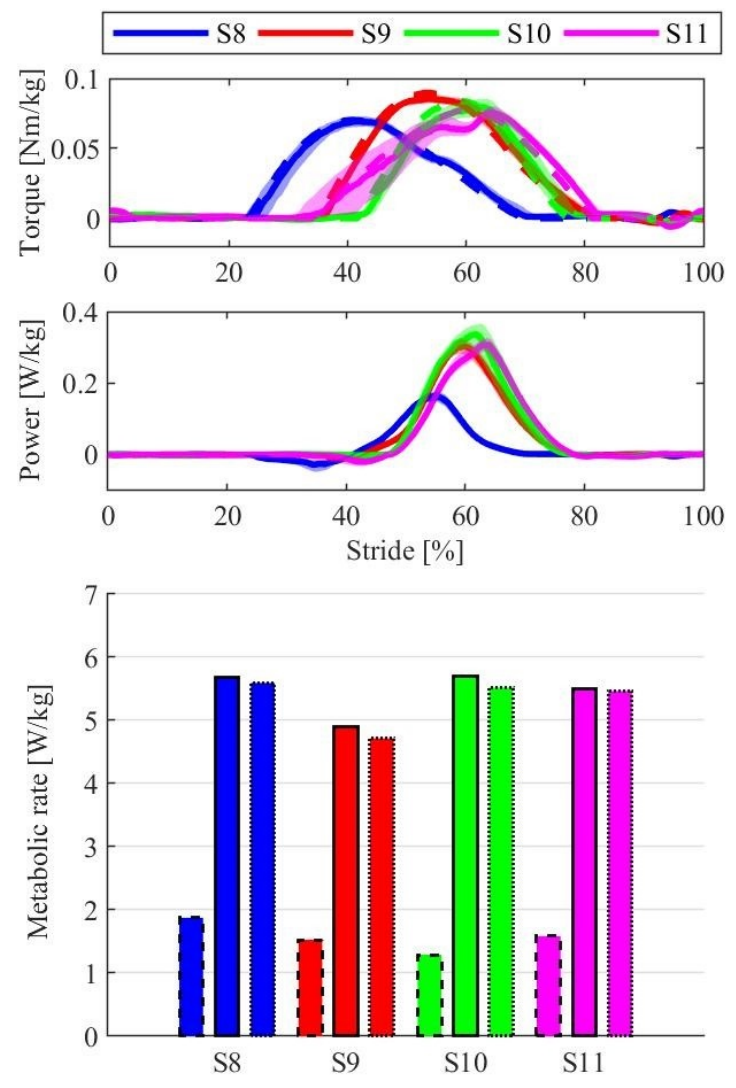

Fig. 5. Torque and power profiles normalized to the subject body mass. In the upper graph, dashed lines represent the commanded averaged profile, whereas the solid line represents the actual delivered torque. The averaged metabolic rate during sitting (dashed contour), the last two minutes of the TM (solid contour) and the AM (dotted contour) conditions for the four subjects. Metabolic rate, paired Student's t-test between TM and AM, $p=0.024<$ 0.05 .

In our previous study, the performance of the gait phase estimator was assessed offline based on capacitive signals [26]. In the present work, a simplified sensing system was used for real-time control of an active exoskeleton. Despite this simplification, the gait phase estimation performance improved in this work, as evidenced by a reduction in $\mathrm{RMSE}_{\phi_{E r r}}$ and $\mathrm{RMSE}_{\phi_{R}}$, which both scored lower than $0.25 \mathrm{rad}$ (4.0\% of the gait cycle), compared to approximately $0.4 \mathrm{rad}(6.4 \%$ of the gait cycle) in our previous work.

The observed performance of the gait phase estimator using C-Sens was slightly worse than that presented in [27], where the exoskeleton hip joint angles (measured in TM) were used to estimate the gait phase using adaptive oscillators. In that study, the $R M S E_{\phi_{R}}$ was lower than $0.09 \mathrm{rad}$ (1.4\% of gait cycle), and the $\mathrm{RMSE}_{\phi_{E r r}}$ was lower than 0.08 (1.3\% of gait cycle - about one third of the errors of the C-Sens-based approach). Notably, in [27] the hip exoskeleton was used only in Transparent Mode; the performance of an assistive controller built on top of the hip angle-based phase estimator would likely have diminished under assistive conditions, due to the different pattern of the angle signals. 
The overall error in the phase estimation in the present study was quantified as $4 \%$ of the gait cycle. Although there is no evidence on the minimum suitable accuracy of a gait phase estimator, a previous study by Ding and colleagues demonstrated that such a value may not lead to different performance of a hip exoskeleton [30].

With the proposed approach, capacitive sensors can reliably obtain information about the user's movement without being affected by the physical human-robot interaction. By comparing the capacitive signals between transparent and assistive modes using the Pearson's correlation coefficient, the advantage of such an architecture was demonstrated: capacitive sensors were significantly less sensitive to the assistive action at normal walking speed ( 0.98 for the capacitive signals against 0.64 for the hip joint angles). Notably, a "de-coupling" of the sensing elements from the robot mechanical structure could be achieved by using other sensors, such as IMUs or sEMG on the shank. However, synchronizing adaptive oscillators to more complex signals may not be straight-forward, particularly if signals (such as raw accelerations gathered from IMUs) have different frequency components. In addition, it should be remarked that the high value of the correlation coefficient of capacitive signals could have been affected by the low-pass filter used to generate the final signals.

Results of $\Delta \phi_{C}$ and $\Delta \phi_{\theta}$ comparisons confirmed that the delivered torque influenced the hip kinematics, with the hip flexion peak occurring earlier in the gait cycle (following heel strike) in AM relative to TM. In assistive mode, the gait phase estimator based on adaptive oscillators would preserve phaselocking with respect to the hip flexion peak, given the inherent adaptation properties of AOs. Nevertheless, the synchrony between the delivered torque and the assisted gait phase would deteriorate, as demonstrated by a difference of about $3 \%$ of the gait cycle of $\Delta \phi_{\theta}$. By tracking the capacitive signal, $\Delta \phi_{C}$ is lower than $2 \%$.

Notably, in this study a pair of sensorized insoles was used as reference gait measurement system to assess the performance of the phase estimator; although insoles are not a gold standard in motion analysis, they have been used in several works and demonstrated suitable performance in recognizing relevant gait events, such as the heel strike [22], [31], [32].

Finally, according to [33], the optimal gait phase to provide peak hip flexion torque is around $60 \%$ of the stride, and results of the present study of the proposed strategy showed that the peak torque was consistently delivered at $58.0 \%$ of the stride. Such assistive action successfully resulted in reduced metabolic cost for all subjects. Even though reduced metabolic rate was observed, it should be considered that the weight of the orthosis itself may have incurred an increased metabolic cost that may partially or entirely offset the assistance provided by the exoskeleton [20]. Notably, the reduction of the metabolic cost was lower with respect to other studies [34]-[37] and this may be partially related to the higher weight of our system and lower assistive positive power. Indeed, the tuning procedure of the assistive profile was driven by the subjective evaluation of comfort which may not be the optimal criteria to achieve high metabolic reductions. Notably, the lack of randomization of the order of experimental conditions and comparison to walking condition without an exoskeleton are two major limitations of this work and will be addressed in future works. Future studies will also investigate the applicability of the proposed approach to users with different types of movement disorders (e.g. muscle weakness).

\section{CONClusion}

This work proposed and validated a novel control architecture for a wearable robot based on capacitive sensing. Capacitive sensors that were easily wearable over users' clothes and robust to variations in cuff placement were used to record relevant biomechanical signals to command the assistive hip flexion-extension torque of a powered hip exoskeleton. The stability of the signal was not affected by the assistive action of the robot, thus confirming the ability of the proposed sensing strategy to maintain high performance in applications with a human user in the control loop of an active device. Moreover, the effectiveness of the strategy was validated by comparing the metabolic cost of walking between assisted and unassisted treadmill walking, thus paving the way to reduce the energetic burden of locomotion in user populations with impaired or limited mobility.

\section{REFERENCES}

[1] G. Colombo, M. Joerg, R. Schreier, and V. Dietz, "Treadmill training of paraplegic patients using a robotic orthosis," Journal of Rehabilitation Research and Development, vol. 37 no. 6, pp. 693, 2000.

[2] J. F. Veneman, R. Kruidhof, E. E. Hekman, R. Ekkelenkamp, E. H. Van Asseldonk, and H. van der Kooij, "Design and evaluation of the LOPES exoskeleton robot for interactive gait rehabilitation," IEEE Transactions on Neural Systems and Rehabilitation Engineering, vol. 15 no. 3, pp. 379-386, 2007.

[3] V. Grosu, C. Rodriguez-Guerrero, S. Grosu, B. Vanderborght, D. Lefeber, "Design of smart modular variable stiffness actuators for robotic-assistive devices," IEEE/ASME Transactions on Mechatronics, vol. 22, no. 4, pp. 1777-1785, 2017.

[4] Y. Feng and Q. Wang, "Combining push-off power and nonlinear damping behaviors for a lightweight motor-driven transtibial prosthesis," IEEE/ASME Transactions on Mechatronics, vol. 22, no. 6, pp. 25122523, 2017.

[5] Y. Lee, Y. Kim, J. Lee, M. Lee, B. Choi, J. Kim, Y. J. Park, and J. Choi, "Biomechanical design of a novel flexible exoskeleton for lower extremities," IEEE/ASME Transactions on Mechatronics, vol. 22, no. 5, pp. 2058-2069, 2017.

[6] M. K. Shepherd and E. J. Rouse, "Design and validation of a torquecontrollable knee exoskeleton for sit-to-stand assistance," IEEE/ASME Transactions on Mechatronics, vol. 22, no. 4, pp. 1695-1704, 2017.

[7] J. L. Pons, "Wearable robots: biomechatronic exoskeletons," John Wiley \& Sons, 2008.

[8] T. Yan, M. Cempini, C. M. Oddo, and N Vitiello, "Review of assistive strategies in powered lower-limb orthoses and exoskeletons," Robotics and Autonomous Systems, vol. 64, pp.120-136, 2015.

[9] J. Rueterbories, E. G. Spaich, B. Larsen, and O. K. Andersen, "Methods for gait event detection and analysis in ambulatory systems," Medical Engineering \& Physics, vol. 32, no. 6, pp. 545-552, 2010.

[10] A. Parri, K. Yuan, D. Marconi, T. Yan, S. Crea, M. Munih, R. M. Lova, N. Vitiello, and Q. Wang, "Real-time hybrid locomotion mode recognition for lower limb wearable robots," IEEE/ASME Transactions on Mechatronics, vol. 22, no. 6, pp. 2480-2491, 2017.

[11] M. R. Tucker, J. Olivier, A. Pagel, H. Bleuler, M. Bouri, O. Lambercy, J. del R. Millan, R. Riener, H. Vallery, and R. Gassert, "Control strategies for active lower extremities prosthetics and orthotics: A review,' Journal of NeuroEngineering and Rehabilitation, vol. 12, no. 1, 2015.

[12] N. d'Elia, F. Vanetti, M. Cempini, G. Pasquini, A. Parri, M. Rabuffetti, ... and N. Vitiello, "Physical human-robot interaction of an active pelvis orthosis: toward ergonomic assessment of wearable robots," Journal of NeuroEngineering and Rehabilitation, 14(1), 29, 2017. 
[13] D. Novak and R. Riener, "A survey of sensors fusion methods in wearable robotics," Robotics and Autonomous System, vol. 73, pp. 155170,2015

[14] C. D. Joshi, U. Lahiri, and N. V. Thakor, "Classification of gait phasesfrom lower limb EMG: Application to exoskeleton orthosis," in Proc. of the IEEE Point-of-Care Healthcare Technologies, 2013, pp. 228-231.

[15] C. L. Lewis and D. P. Ferris, "Invariant hip moment pattern while walking with a robotic hip exoskeleton," Journal of Biomechanics, vol. 44 , no. 5 , pp. $789-793,2011$

[16] T. Lenzi, M. C. Carrozza, and S. K. Agrawal, "Powered hip exoskeletons can reduce the user's hip and ankle muscle activations during walking," IEEE Transactions on Neural Systems and Rehabilitation Engineering, vol. 21, no. 6, pp. 938-948, 2013.

[17] J. Taborri, E. Palermo, S. Rossi, and P. Cappa, "Gait partitioning methods: A systematic review," Sensors, vol. 16, no. 1, pp. 66, 2016.

[18] Z. Yang, and C. Harrison, "Tomo: Wearable, low-cost electrical impedance tomography for hand gesture recognition," Proceedings of the 28th Annual ACM Symposium on User Interface Software \& Technology, 2015.

[19] M. Boutaayamou, C. Schwartz, J. Stamatakis, V. Denoel, D. Maquet, B. Forthomme, J. Croisier, B. Macq, J. G. Verly, G. Garraux, and O. Bruls "Development and validation of an accelerometer-based method for quantifying gait events," Medical Engineering \& Physics, vol. 37, no. 2, pp. 226-232, 2015.

[20] V. R. Garate, A. Parri, T. Yan, M. Munih, R. M. Lova, N. Vitiello, and R. Ronsse, "Walking assistance using artificial primitives: A novel bioinspired framework using motor primitives for locomotion assistance through a wearable cooperative exoskeleton," IEEE Robotics and Automation Magazine, vol. 23, no. 1, pp. 83-95, 2016.

[21] D. Kotiadis, H. J. Hermens, and P. H. Veltink, 'Inertial gait phase detection for control of a drop foot stimulator: Inertial sensing for gait phase detection," Medical Engineering \& Physics, vol. 32, no. 4, pp. 287-297, 2010.

[22] S. Crea, M. Donati, S. M. M. De Rossi, C. M. Oddo, and N. Vitiello, "A wireless flexible sensorized insoles for gait analysis," Sensors, vol. 14, pp. 1073-1093, 2014

[23] E. Zheng, L. Wang, K. Wei, and Q. Wang, "A noncontact capacitive sensing system for recognizing locomotion modes of transtibial amputees," IEEE Transactions on Biomedical Engineering, vol. 61, no. 12, pp. 2911-2920, 2014

[24] K. Kong and D. Jeon, "Design and Control of an Exoskeleton for the Elderly and Patients," IEEE/ASME Transactions on Mechatronics, vol. 11, no. 4, 2006.

[25] F. Giovacchini, F. Vannetti, M. Fantozzi, M. Cempini, M. Cortese, A. Parri, T. Yan, D. Lefeber, and N.Vitiello, "A light-weight active orthosis for hip movement assistance," Robotics and Autonomous Systems, vol. 73, pp. 123-134, 2015.

[26] E. Zheng, S. Manca, T. Yan, A. Parri, N. Vitiello, and Q. Wang, "Gait phase estimation based on noncontact capacitive sensing and adaptive oscillators," IEEE Transactions on Biomedical Engineering, vol. 64, no. 10, pp. 2419-2430, 2017

[27] T. Yan, A. Parri, V. R. Garate, M. Cempini, R. Ronsse, and N. Vitiello, "An oscillator-based smooth real-time estimate of gait phase for wearable robotics," Autonomous Robots, vol. 41, no. 3, pp. 759-774, 2017.

[28] G. A. Pratt and M. M. Williamson, "Series elastic actuators," in Proc. of the IEEE/RSJ International Conference on Intelligent Robots and Systems, 1995, pp. 399-406.

[29] M. Donati, N. Vitiello, S. M. M. De Rossi, T. Lenzi, S. Crea, A. Persichetti, F. Giovacchini, B. Koopman, J. Podobnik, M. Munih, and M. C. Carrozza, "A flexible sensor technology for the distributed measurement of interaction pressure," Sensors, vol. 13, no. 1, pp. 10211045,2013

[30] Y. Ding, F. A. Panizzolo, C. Siviy, P. Malcolm, I. Galiana, K. G. Holt, and C. J. Walsh, "Effect of timing of hip extension assistance during loaded walking with a soft exosuit," Journal of NeuroEngineering and Rehabilitation, vol. 13, no. 1, pp. 87, 2016.

[31] S. M. M. De Rossi, S. Crea, M. Donati, P. Reber?ek, D. Novak, N. Vitiello, T. Lenzi, J. Podobnik, M. Munih, and M. C. Carrozza, "Gait segmentation using bipedal foot pressure patterns," in Proc. Of the IEEE Biomedical Robotics and Biomechatronics conference, 2012.

[32] S. Crea, B. B. Edin, K. Knaepen, R. Meeusen, and N. Vitiello, "Timediscrete vibrotactile feedback contributes to improved gait symmetry in patients with lower limb amputations: Case series," Physical Therapy, vol. 97 , no. 2, pp. 198-207, 2017.
[33] A. J. Young, J. Foss, H. Gannon, and D. P. Ferris, 'Influence of power delivery timing on the energetic and biomechanics of humans wearing a hip exoskeleton," Frontiers in Bioengineering and Biotechnology, vol.5, no. $4,2017$.

[34] F. A. Panizzolo, I. Galiana, A. T. Asbeck, C. Siviy, K. Schmidt, K. G. Holt, and C. J. Walsh, "A biologically-inspired multi-joint soft exosuit that can reduce the energy cost of loaded walking," Journal of NeuroEngineering and Rehabilitation, vol. 13, no. 1, 2016.

[35] A. T. Asbeck, R. J. Dyer, A. F. Larusson, and C. J. Walsh, "Biologicallyinspired soft exosuit," in Proc. of the IEEE International Conference on Rehabilitation Robotics, 2013.

[36] B. T. Quinlivan, S. Lee, P. Malcolm, D. M. Rossi, M. Grimmer, C. Siviy, N. Karavas, D. Wagner, A. Asbeck, I. Galiana, and C. J. Walsh, "Assistance magnitude versus metabolic cost reductions for a tethered multiarticular soft exosuit," Science Robotics, vol. 2, no. 2, 2017.

[37] P. Malcolm, S. Lee, S. Crea, C. Siviy, F. Saucedo, I. Galiana, F A. Panizzolo, K. G. Holt, and C. J. Walsh, "Varying negative work assistance at the ankle with a soft exosuit during loaded walking," Journal of NeuroEngineering and Rehabilitation, vol. 14, no. 1, 2017.

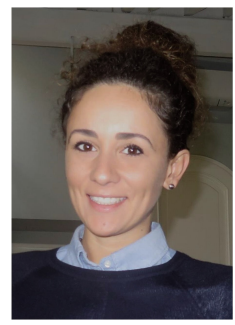

Simona Crea received her $\mathrm{PhD}$ in Biorobotics from Scuola Superiore Sant'Anna on December 2015. Since April 2017 she is an Assistant Professor at The BioRobotics Institute of Scuola Superiore Sant'Anna, where she works on wearable robotics. Her research activities are focused on developing and validating novel wearable technologies and paradigms of human-robot interaction. She is project manager of the H2020 CYBERLEGs Plus Plus project, and co-PI of two national projects funded by INAIL (the National Institute for Insurance against

Accidents at Work).

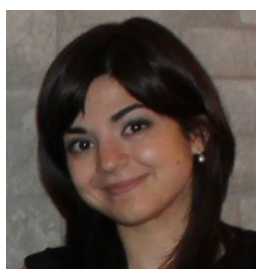

Silvia Manca received the B. Sc. and the M. Sc. in Biomedical Engineering from the University of Pisa, Italy, respectively in 2012 and 2015. In 2014 she had joined the BioRobotics Institute where she conducted research activities in the wearable robotic$\mathrm{s}$ field for almost three years. Her research topic was focused in the development of adaptive strategies to control lower limb wearable robots. From 2017 she is employed in the private industry where she is in role of developing, testing and integrating software application for real-time systems.

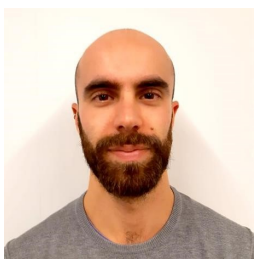

Andrea Parri received the M.Sc. degree in Biomedical Engineering (cum laude) from the University of Pisa in 2014. He received the $\mathrm{PhD}$ in BioRobotics from Scuola Superiore Sant'Anna in June 2018. Since December 2018 he is a R\&D employee of IUVO s.r.l., spin-off company of Scuola Superiore Sant'Anna. Thanks to the strong expertise matured in the academic field, his interests are focused on the design and development of adaptive control architectures for wearable robots and their validation with target end-users in in-lab and ecological settings. 


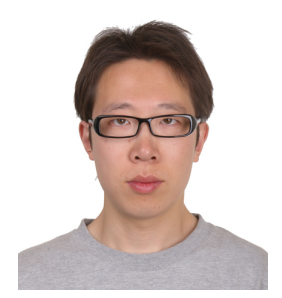

Enhao Zheng (M'16) received his B.E degree from Xidian University, Xian, China, in 2010 and Ph.D. degree in dynamics and control from Peking University, Beijing, China, in 2016. He is currently an Associate Professor in The State Key Laboratory of Management and Control for Complex Systems, Institute of Automation, Chinese Academy of Sciences. His research interests include human-machine interface and wearable robotics.

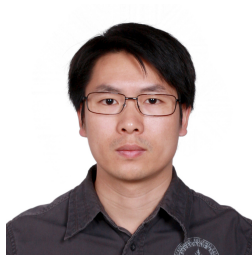

Jingeng Mai received his B.E degree from China University of Geosciences (Beijing) in 2005 and M.Sc. degree from Peking University in 2008. After receiving $\mathrm{Ph} . \mathrm{D}$. degree from Beihang University in 2016, he joins Peking University as postdoctoral researcher. He is currently an Assistant Professor in the College of Engineering, Peking University, Beijing, China. His research interests include intelligent manufacturing and robotics.

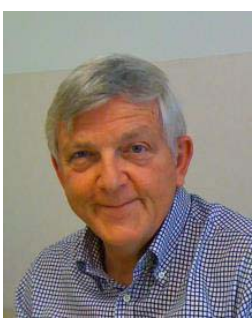

Raffaele Molino Lova is a Cardiologist and Cardiovascular Surgeon and has been involved for many years in the clinical management of the Cardiac Rehabilitation Unit of the Fondazione Don Gnocchi (Florence, Italy), where are admitted patients recently undergone cardiac surgery. His main research interest is in the field of exercise physiology, gas exchange kinetics, energy cost of walking and robotic systems to assist people with lower-limb movement impairment. He is co-author of more than 80 ISI peer-reviewed papers and has been acknowledged as

Full Professor in Rehabilitation Medicine by the Italian Ministry of Education on a national basis.

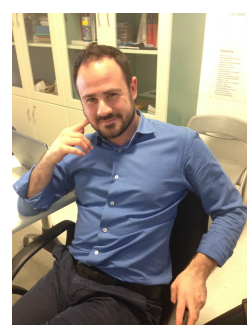

Nicola Vitiello is Associate Professor with The BioRobotics Institute (Scuola Superiore SantAnna, SSSA, Pisa, Italy) where he leads the Wearable Robotics Laboratory. He is co-author of more than 70 ISI/Scopus papers and co-inventor of more than 20 patents/patent applications. He served as the Scientific Secretary of the EU FP7 CA-RoboCom project, and he was the scientific coordinator of the EU FP7 CYBERLEGs project. Currently he is the scientific coordinator of the H2020-ICTCYBERLEGs Plus Plus project, the national project MOTU funded by INAIL and is partner of the H2020-FoF-HUMAN project. $\mathrm{He}$ is co-founder, director and advisor of IUVO Srl, a spin-off company of SSSA.

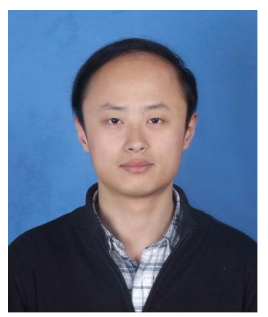

Qining Wang (S'06-M'09-SM'19) received the Ph.D. degree in dynamics and control from Peking University, Beijing, China, in 2009. He is currently an Associate Professor in the College of Engineering, Peking University, China. He serves as the Vice-Director of the Center for Robotics in Peking University, the Director of the Center for Rehabilitation Engineering in Peking University and the Director of the Beijing Engineering Research Center of Intelligent Rehabilitation Engineering. He has authored/coauthored over 160 scientific papers in international journals and refereed conference proceedings. His research interests include bio-inspired robots and rehabilitation robotics. Dr. Wang received the Best Technical Paper Award at the 17th International Conference on Climbing and Walking Robots (CLAWAR) in 2015 and the 18th International Conference on CLAWAR in 2016. He serves as an Advisor of the IEEERAS Technical Committee on Wearable Robotics, an Associate Editor for the IEEE Robotics \& Automation Magazine (2016-2018), a Technical Editor for the IEEE/ASME Trans. Mechatronics (2017-), an Associate Editor for the journal Robotica (2018-) and an Associate Editor for the IEEE Trans. Medical Robotics and Bionics (2018-). 